\title{
Do we really need postoperative ICU management after elective surgery? No, not any more!
}

\author{
Paolo Taccone ${ }^{1 *} \mathbb{D}$, Thomas Langer ${ }^{2}$ and Giacomo Grasselli ${ }^{1}$
}

(๑) 2017 Springer-Verlag Berlin Heidelberg and ESICM

A growing amount of evidence shows that postoperative complications are a dramatically relevant health issue estimated to cause 3-12 million deaths every year [1], resulting in one of the leading causes of mortality, even more lethal than road accidents or diabetes [2]. Hence, it is now acknowledged that more attention is needed to prevent, recognize early and treat any potentially lifethreatening complication that might occur during the postoperative period. Accordingly, ICU admission of high-risk surgical patients has been historically regarded as of crucial importance to reduce the risk of perioperative death. However, with the increasing request for safe and effective medical provisions, a cost-efficacy analysis is mandatory to achieve these goals while avoiding any waste of the limited resources available, especially in low-income countries. Recently, the value of routine ICU admission after elective surgery has been questioned, given its costs and the lack of robust evidence supporting it $[3,4]$. Moreover, identification of patients at highest risk, who might benefit from ICU admission, remains a major challenge leading to unacceptable inequity in resource distribution [5].

To this end, the recent article by Kahan et al. [6] et al. provides important elements to answer one of the most relevant questions for anesthesiologists, surgeons, and intensivists working in the perioperative field: do we really need to admit high-risk surgical patients to the ICU after an elective procedure? In their article, the authors report the results of a secondary analysis of the

\footnotetext{
*Correspondence: paolo.taccone@policlinico.mi.it

${ }^{1}$ Department of Anesthesia, Critical Care and Emergency, Fondazione IRCCS Ca' Granda, Ospedale Maggiore Policlinico, Via F. Sforza 35, 20122 Milan, Italy

Full author information is available at the end of the article
}

International Surgical Outcomes Study (ISOS) database [7]: in a large cohort of more than 44,000 patients from 474 hospitals in 27 countries, no direct association was found between perioperative mortality and ICU utilization (expressed as number of ICU beds available and rate of patients admitted in ICUs after surgery). Some relevant limitations of this study need to be underlined: (a) the non-randomized nature of the study; (b) the potential effect of unmeasured confounding factors on outcome analysis; (c) the lack of distinction between pre-planned "elective" ICU admissions, for monitoring of high-risk patients, and unplanned "urgent" admissions, for intensive care support after major intraoperative complications (e.g., severe bleeding); (d) the limited external validity due both to the simplistic "ICU" definition (i.e., facilities with overnight mechanical ventilation capabilities) and to the patient enrollment criteria (in particular, since only elective surgery was considered, the results might not be transferable to the emergency setting). In spite of these biases, the large population prospectively studied and the high number of participating centers around the world offer a unique opportunity to investigate the utility of routine ICU admission after elective surgery.

Many factors may explain the lack of efficacy showed by Kahan et al.s study. First, most patients admitted postoperatively to the ICU spent there only 1 or 2 days, while many complications (and related deaths) actually occur later during the hospital stay, i.e., when the patient has already been transferred to the general ward. Second, the concept that ICU admission may improve long-term outcome by correcting early physiological derangements related to the surgical stress (e.g., hypothermia, fluid imbalances, postoperative residual paralysis, etc.) might be less valid today, since surgical procedures are

\section{实 Springer}


becoming less invasive and anesthesia practice is continuously improving. Consequently, the ICU stay could paradoxically be more harmful than beneficial for elective surgical patients because of potential side effects, such as increased risk of nosocomial infections, reduced access to early mobilization, oversedation, sleep deprivation, stress, and delirium.

Thus, since most patients admitted electively to the ICU do not have organ failures and only need close cardiorespiratory and metabolic monitoring, it is possible that this level of assistance might be adequately delivered in specialty wards with improved nurse staffing and advanced monitoring modalities (e.g., telemetry). We should therefore change our beliefs on the role of standard ICU in the postoperative period, and recognize that alternative organizational models might provide a more efficient assistance to surgical patients. A postoperative "intermediate care" ward may represent a sufficiently safe environment for the majority of surgical patients, with a significant reduction in costs and ICU-related side effects. The resource-savings benefits of such a model may allow us to guarantee appropriate assistance to a larger number of patients, avoiding the selection biases related to unreliable preoperative risk assessment and unstandardized patient triage. Moreover, patient monitoring could be prolonged beyond the first postoperative days, when late complications (e.g., infections) are more likely to occur and are frequently under-recognized and undertreated.

Therefore, what should we do in everyday clinical practice when facing our next "high-risk" surgical patient scheduled for an elective surgical procedure? If we work in a well-organized hospital with high-quality wards and availability of other pivotal services (e.g., acute pain service, rapid response team, etc.), elective ICU admission is presumably not needed. And what organizational interventions should we aim for to improve postoperative care in our institutions? Well, it is time for us to abandon the assumption that dedicated postoperative ICU facilities and/or more ICU beds can directly reduce mortality in the surgical population, and we should start to reallocate our resources and efforts in other steps of patient assistance.
In conclusion, it is a clear priority for perioperative medicine to find more efficient alternatives to traditional ICUs for postoperative care of elective surgical patients. Local and global quality-improving initiatives are certainly needed to standardize and implement postoperative care in a safe and cost-effective manner.

\section{Author details}

${ }^{1}$ Department of Anesthesia, Critical Care and Emergency, Fondazione IRCCS Ca'Granda, Ospedale Maggiore Policlinico, Via F. Sforza 35, 20122 Milan, Italy.

${ }^{2}$ Department of Pathophysiology and Transplantation, University of Milan,

Milan, Italy.

\section{Compliance with ethical standards}

Conflicts of interest

All the authors declare that they have no conflict of interest.

Received: 25 March 2017 Accepted: 21 April 2017

Published online: 18 May 2017

\section{References}

1. Pearse RM, Moreno RP, Bauer P, Pelosi P, Metniz P, Spies C, Vallet B, Vincent $J$, Hoeft A, Rhodes A (2012) Mortality after surgery in Europe: 7 day cohort study. Lancet 380(9847):1059-1065

2. World Health Organization (2017) The top 10 causes of death. http:// www.who.int/mediacentre/factsheets/fs310/en/. Accessed 16 March 2017

3. Wunsch H, Gershengorn HB, Cooke CR, Guerra C, Angus DC, Rowe JW, Li $\mathrm{G}$ (2016) Use of intensive care services for Medicare beneficiaries undergoing major surgical procedures. Anesthesiology 124:899-907

4. Gillies MA, Pearse RM (2016) Intensive care after high-risk surgery: what's in a name? Anesthesiology 124:761-762

5. Gillies MA, Harrison EM, Pearse RM et al (2017) Intensive care utilization and outcomes after high-risk surgery in Scotland: a population-based cohort study. Br J Anaesth 118(1):123-131

6. Kahan BC, Koulenti D, Arvaniti Ket al (2017) Relationship between critical care provision and mortality following elective surgery: prospective analysis of data from 27 countries. Intensive Care Med. doi:10.1007/ s00134-016-4633-8

7. The International Surgical Outcomes Study Group (2016) Global patient outcomes after elective surgery: prospective cohort study in 27 low-, middle- and high-income countries. Br J Anaesth 117(5):601-609 